\title{
SIMULTANEOUS STATE AND PARAMETER ESTIMATION BASED ACTUATOR FAULT DETECTION AND DIAGNOSIS FOR AN UNMANNED HELICOPTER
}

\author{
$\mathrm{CHONG}_{\mathrm{WU}}{ }^{a, b}, \mathrm{JUNTONG}^{a, *}, \mathrm{DAlei} \mathrm{SONG}^{a}, \mathrm{XIN}^{a, b}, \mathrm{JIANDA} \mathrm{HAN}^{a}$ \\ ${ }^{a}$ State Key Laboratory of Robotics, Shenyang Institute of Automation \\ Chinese Academy of Sciences, No. 114 Nanta Street, Shenyang, Liaoning Province, China \\ e-mail: \{wuchong, qijt, qixin, daleisong, jdhan\} @sia.cn \\ ${ }^{b}$ University of Chinese Academy of Sciences, No. 19A Yuquan Road, Beijing, China
}

\begin{abstract}
Simultaneous state and parameter estimation based actuator fault detection and diagnosis (FDD) for single-rotor unmanned helicopters (UHs) is investigated in this paper. A literature review of actuator FDD for UHs is given firstly. Based on actuator healthy coefficients (AHCs), which are introduced to represent actuator faults, a combined dynamic model is established with the augmented state containing both the flight state and AHCs. Then the actuator fault detection and diagnosis problem is transformed into a general nonlinear estimation one: given control inputs and the measured flight state contaminated by measurement noises, estimate both the flight state and AHCs recursively in each time-step, which is also known as the simultaneous state and parameter estimation problem. The estimated AHCs can further be used for fault tolerant control (FTC). Based on the existing widely used nonlinear estimation methods such as the unscented Kalman filter (UKF) and the extended set-membership filter (ESMF), three kinds of adaptive schemes (KF-UKF, MIT-UKF and MIT-ESMF) are proposed by our team to improve the actuator FDD performance. A comprehensive comparative study on these different estimation methods is given in detail to illustrate their advantages and disadvantages when applied to unmanned helicopter actuator FDD.
\end{abstract}

Keywords: actuator fault detection and diagnosis, unmanned helicopter, Kalman filter, set-membership filter, adaptive scheme.

\section{Introduction}

Helicopters have been widely used in both civilian and military fields due to their capabilities of hovering, vertical take-off and landing, low-altitude and low-speed flight. In the past two decades, the unmanned helicopter (UH) has become an attractive research topic in academic communities worldwide and numerous research groups have designed their own unmanned helicopter platforms such as Yamaha-RMAX of Carnegie Mellon University (Amidi et al., 1998), GTMax of the Georgia Institute of Technology (Johnson and Schrage, 2003), ServoHeli of the Shenyang Institute of Automation, Chinese Academy of Sciences (Qi et al., 2009) and the Lion unmanned aerial vehicle (UAV) of the National University of Singapore (Cai et al., 2011b).

The increasing utilization of UHs in civilian environment demands higher reliability and safety to

\footnotetext{
*Corresponding author
}

avoid potential accidents. However, structure features of the helicopter induce that it does not have the same graceful degradation property as other aerial vehicles, such as fixed-wing aircraft and airships, under faults (degradation) or failures (out of order) (Heredia et al., 2008). Furthermore, the normally small size, light weight and compact structure characteristics of UHs make the redundancy of on-board sensors and actuators redundancy extremely limited. As is known, the main rotor of the helicopter is used not only to provide the lift but also to control the helicopter. In order to control the main rotor, a swashplate is used. A small UH's swashplate, which mainly performs the lateral, longitudinal and vertical motion to control the $\mathrm{UH}$ through three actuators, is illustrated in Fig. 1. There is no redundancy among these actuators and the actuator fault will directly lead to malfunction of the swashplate.

In this paper, actuator faults of UHs are considered. Typically, actuator faults mainly include constant output 


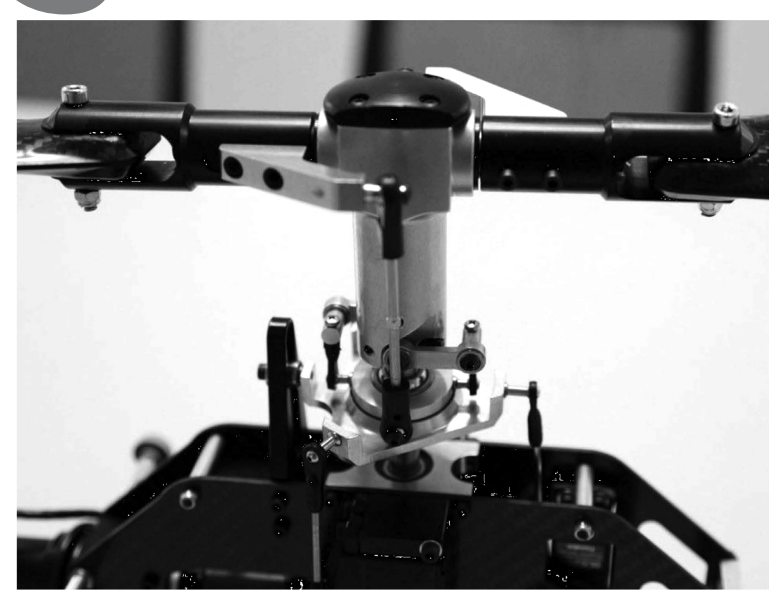

Fig. 1. Small UH's swashplate with three actuators.

faults, constant gain change faults and drift faults. A constant output faulty means that the fault actuator cannot respond to the control input and will stay at a fixed position. Typical reasons for constant output faults of UHs are servo stucks and engine failures. A constant gain change fault represents the fact that the real output value of fault actuator is proportional to the fault-free case. Servo power and engine power lost are the representatives. A drift fault means that the actuator's output value changes along with the flight state of the UH (Qi et al., 2014). From another point of view, Heredia et al. (2004) classify actuator faults according to the location of the actuators and whether or not they have been stuck: (i) the servo involved in the rolling (or pitching) motion has a fault, but does not get stuck, (ii) the servo involved in the rolling (or pitching) motion actually gets stuck, so neither the collective nor the rolling (or pitching) actuators will work, (iii) the collective actuator can no longer work or it may work with a limited range, due to a fault in the mechanical links. The first two kinds of faults are investigated in this paper.

In order to achieve acceptable performance of the post-fault UH system, the first task is to obtain fault information in time, because it is the basis to reconfigure the control strategy or mission planning to guarantee system stability. Many fault detection and diagnosis (FDD) techniques have been proposed to obtain fault information, and these approaches have been widely used in the process industry. However, research results of FDD approaches used for UHs are limited, especially for actuator FDD. Generally speaking, based on the amount of fault information provided, FDD approaches can be divided into three levels: fault detection, fault isolation and fault identification. Fault detection is just to decide whether or not a fault has occurred, fault isolation is to determine the location of the fault and its type, and fault identification is to determine the magnitude of the fault
(Zhang and Jiang, 2008; Qi et al., 2013). Clearly, the higher the level, the more detailed information can be provided. On the other hand, FDD techniques can also be classified into model based and model free approaches. Compared with model free approaches, model based approaches can provide more details of the fault, which can usually cover all the three levels of FDD. Thus, most existing approaches for UHs' actuator FDD are model based. The key idea of these approaches is to generate a residue according to outputs of a real system and the known model. Based on the way of generating the residue, the FDD approaches can be classified into (i) parity space based methods, (ii) observer based methods (Heredia et al., 2004; 2008; Arne and Jürgen, 2011), (iii) estimation based methods (Ducard and Geering, 2008; Qi et al., 2007; Campbell et al., 2007).

Two ways were utilized by Drozeski et al. (2005) for UH actuator fault detection. The first one is the state-dependent approach, which is based on neural network training and used for collective pitch control actuator fault detection. Another one is the sensor-dependent approach, which employs sensors placed on the actuators to detect a fault; this approach is more preferable if sensor mounting is feasible. The authors provided the first real flight test validation of swashplate actuator fault detection and fault tolerant control.

Heredia et al. $(2004 ; 2008)$ used a Luenberger observer based on an input-output model for actuator fault detection. The input-output ARX model is identified based on the collected input and output data of the UH in a fault-free case, then the fault-free output can be predicted based on the input-output model. The corresponding residue is described by the deviation from measured output to nominal output:

$R(k)=\sum_{i=1}^{3} m_{i}\left(v_{i}(k)-\hat{v}_{i}(k)\right)^{2}+\sum_{i=1}^{3} n_{i}\left(\omega_{i}(k)-\hat{\omega}_{i}(k)\right)^{2}$,

where $v_{i}$ and $\omega_{i}$ are measured linear and angular velocities, respectively, $\hat{v}_{i}$ and $\hat{\omega}_{i}$ are nominal linear and angular velocities, respectively, $m_{i}$ and $n_{i}$ are weighting factors. In order to achieve fault isolation, an independent residue is constructed for each different actuator fault, and a reasoning method is used for residue evaluation.

A robust fault isolation observer is proposed by Arne and Jürgen (2011) for actuator fault detection. The target is to obtain the transfer function matrix $G_{r f}(s)$ associating faults and residues,

$$
G_{r f}(s)=\operatorname{diag}\left(g_{r_{1} f_{1}}(s), \ldots, g_{r_{n_{f}} f_{n_{f}}}(s)\right)
$$

where $g_{r_{i} f_{i}}(s)$ is the transfer function from fault $f_{i}$ to 
residue $r_{i}$. The residue $r$ is generated by the fault observer

$$
\left\{\begin{array}{l}
\dot{\hat{x}}(t)=A \hat{x}(t)+B u(t)+L(y(t)-C \hat{x}(t)), \\
r(t)=V(y(t)-C \hat{x}(t)),
\end{array}\right.
$$

where $L$ and $V$ are observer gains which should be designed for the specific problem. This method is capable of isolating simultaneous actuator and sensor faults.

Compared with observer based approaches, estimation based approaches have been investigated more extensively. These approaches can be classified into two groups: parameter estimation approaches and simultaneous state and parameter estimation approaches (Zhang and Jiang, 2008). The latter have attracted more attention in recent years. There are two key issues involved in estimation based approaches: the actuator fault's mathematical model and estimation method. Qi et al. (2007) proposed an actuator fault model which uses actuator healthy coefficients (AHCs) to represent various UH actuator faults. Amoozgar et al. (2013) set forth an additive and multiplicative actuator fault model established for four rotors of a quadrotor unmanned aerial vehicle (UAV). Apart from that, a locked-in-place and floating actuator mathematical fault model for a fix-wing UAV were defined by Ducard and Geering (2008).

Taken the actuator fault parameter and flight state as an augmented state, the actuator FDD problem can be transformed into a general nonlinear state estimation one, which is also called the simultaneous state and parameter estimation problem. Many common nonlinear estimation methods have been utilized for such a simultaneous state and parameter estimation problem, with proper selection of the augmented state's noise characteristics. In the work of Ducard and Geering (2008), locked-in-place and floating actuator faults are estimated by an extended Kalman filter (EKF) for a fix-wing UAV. Campbell et al. (2007) used a square root sigma point filter and a square root extended set-membership filter (ESMF) for fault estimation on for fixed-wing UAVs. Many comparisons have already been made for these methods (Zhou and Han, 2007; Bätz et al., 2013; Cui et al., 2005).

Even though the simultaneous state and parameter estimation problem can be considered a general nonlinear estimation one, it differs from other estimation problems in that the actuator fault's parameters have constant values normally, but with abrupt changes as actuator faults are encountered. The characteristics of actuator fault parameter noise are quite different between the normal case and the fault case, which means a constant noise characteristic setting will be either overestimated in the normal case or underestimated in the fault case. An adaptive noise characteristics setting scheme should be introduced to improve the estimation accuracy. Based on the unscented Kalman filter (UKF), which is believed to yield a more accurate estimation compared with the frequently used EKF (Kotecha and Djuric, 2003), two adaptive updating methods were proposed: the MIT-rule based updating method (MIT-UKF) (Qi et al., 2007) and the KF assist updating method (KF-UKF) (Qi et al., 2012). An MIT-rule based adaptive extended set-membership filter (MIT-ESMF) was also proposed by $\mathrm{Wu}$ et al. (2012), which assumes the system and measurement noise signals as unknown but bounded to better fit real applications. Xiong (2013) established another set-membership filter with an emphasis on the system model's uncertainty using interval analysis (the model uncertainty is assumed to be unknown but bounded, which is more suitable for the real case where modeling is inevitably inaccurate). Since simultaneous state and fault parameter estimation based FDD is a newly developed research area, a comparative study will be given in this paper regarding the different estimation methods to illustrate their advantages and disadvantages for actuator FDD on a UH, with emphasis on KF-UKF, MIT-UKF and MIT-ESMF.

The paper is organized as follow. Section 2 contains the overall scheme of simultaneous state and parameter estimation based actuator FDD, along with the AHC fault model. In Section 3, a complete nonlinear unmanned helicopter dynamic model is introduced as the basis for estimation. Section 4 briefly introduces the existing estimation methods that can be used for simultaneous state and parameter estimation. A comparative study of these methods is given in Section 5 followed with a conclusion in Section 6 .

\section{Problem statement of simultaneous state and parameter estimation based actuator fault detection and diagnosis for a $\mathbf{U H}$}

The overall scheme of actuator FDD is illustrated in Fig.2. The main difference of this scheme when compared with the normal flight control system is that the actuator's fault model is explicitly used to represent the actuator fault. Through the combination of the UH's dynamic model and the actuator's fault model, a combined model can be established with an augmented state of the normal flight state and AHCs. Then the AHCs can be estimated on-line along with the flight state, given the control input provided by the flight controller and the measurement obtained from the on-board sensors. With the estimated AHCs, the flight controller can adjust its structure or coefficients to compensate for the actuator fault so as to ensure system stability. In this paper, the main focus will be on simultaneous state and AHCs estimation methods; fault tolerant control (FTC) design can be found in the works (Qi et al., 2007; Campbell et al., 2007).

The general dynamic model of the UH is defined as 


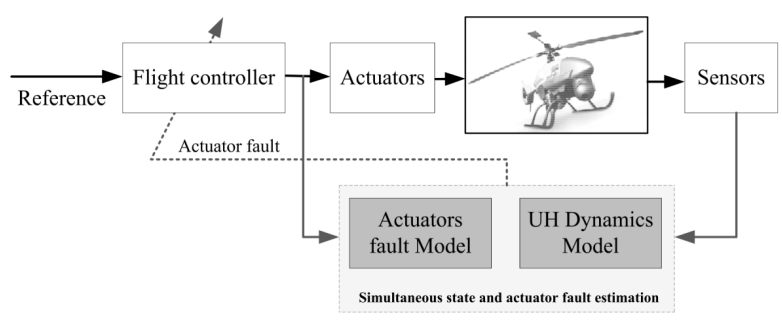

Fig. 2. Actuator fault detection and identification scheme.

follows:

$$
\left\{\begin{array}{l}
x_{k+1}=f\left(x_{k}, u_{k}\right)+\omega_{k}, \\
y_{k}=h\left(x_{k}\right)+v_{k},
\end{array}\right.
$$

where $x_{k}$ is the UH's flight state vector, $u_{k}$ is the actuator's control input, and $y_{k}$ is the measurement obtained by the on-board sensors.

In the normal case, the actuator's real action $\hat{u}_{k}$ is in accordance with the expected actuator action $u_{k}$. But $\hat{u}_{k}$ will deviate from $u_{k}$ in the presence of various actuator faults. The mathematical model of an actuator fault is defined as

$$
\hat{u}_{k}=g\left(\theta_{k}, u_{k}\right),
$$

where $\theta_{k}$ is the parameter vector of the actuator fault and can generally be assumed as a random walk variable (Bian et al., 2011)

$$
\theta_{k+1}=\theta_{k}+\omega_{\theta, k},
$$

where $\omega_{\theta, k}$ represents the zero-mean process noise.

Then the combined dynamic model of UH and actuator fault can be represented as

$$
\left\{\begin{array}{l}
x_{k+1}=\hat{f}\left(x_{k}, \theta_{k}, u_{k}\right)+\omega_{k}, \\
\hat{f}\left(x_{k}, \theta_{k}, u_{k}\right)=f\left(x_{k}, g\left(\theta_{k}, u_{k}\right)\right), \\
\theta_{k+1}=\theta_{k}+\omega_{\theta, k}, \\
y_{k}=h\left(x_{k}\right)+v_{k} .
\end{array}\right.
$$

The actuator FDD problem can be transformed into a nonlinear state estimation one by defining the augmented system state vector $z_{k}=\left[x_{k}, \theta_{k}\right]^{T}$. The two key issues involved are the actuator fault's mathematical model and the simultaneous state and parameter estimation method. The actuator fault's mathematical model will be introduced in this section, and the estimation methods will be discussed in Section 4.

Generally, a UH is governed by five control surfaces to perform six-degrees-of-freedom movements (Cai et al., 2011b): the throttle servo $\theta_{\text {throttle }}$ for the main rotor rotation speed control, the collective pitch servo $\theta_{M}$ for main rotor blade angle control, the aileron servo $\theta_{\text {Lat }}$ for lateral motion control, the elevator servo $\theta_{\text {lon }}$ for longitudinal motion control and the rudder servo $\theta_{T}$ for tail rotor blade angle control. Normally, the rotation speed of the main rotor is kept constant using a governor by $\theta_{\text {throttle }}$ to facilitate the controller design. Obviously, the $\mathrm{UH}$ is an under-actuated system and the main control input is $\left[\theta_{M}, \theta_{\text {lat }}, \theta_{\text {lon }}, \theta_{T}\right]$.

Actuator faults of UHs mainly include constant output faults, constant gain change faults and drift faults. For explicit presentation of an actuator fault, an actuator fault model is established as follows (Qi et al., 2006):

$$
\left\{\begin{array}{l}
\hat{u}_{k}=\Gamma_{f} u_{k}+\Delta_{f}, \\
\Gamma_{f}=\operatorname{diag}\left[\gamma_{1}, \gamma_{2}, \ldots, \gamma_{l}\right], \\
\Delta_{f}=\operatorname{diag}\left[\delta_{1}, \delta_{2}, \ldots, \delta_{l}\right],
\end{array}\right.
$$

where $\gamma_{i}$ and $\delta_{i}$ are the proportional effectiveness and fault biases of the $i$-th actuator, respectively, and $\theta_{k}=$ $\left[\gamma_{1}, \ldots, \gamma_{l}, \delta_{1}, \ldots, \delta_{l}\right]$ are defined as AHCs.

With this actuator fault model, various actuator faults can be expressed effectively as follows:

- constant output faults: $\gamma_{i}=0, \delta_{i}=k$,

- constant gain change faults: $\gamma_{i}=k, \delta_{i}=0$,

- drift faults: $\gamma_{i}=\gamma(t), \delta_{i}=\delta(t)$.

\section{Dynamic model of the UH}

As the basis of state estimation, a UH's dynamic model will be introduced in this section. Through a combination with the actuator fault model introduced in Section 2 a combined dynamic model can be established for the nonlinear augmented state estimation.

The single rotor unmanned helicopter generally consists of the main rotor, the tail rotor, the fuselage, the horizontal stabilizer and the vertical stabilizer. For simplification, the unmanned helicopter is considered as a rigid body with external forces and torques generated respectively by the main rotor, tail rotor and fuselage. The body frame is defined as shown in Fig. 3 ( $\mathrm{He}$ and Han, 2010).

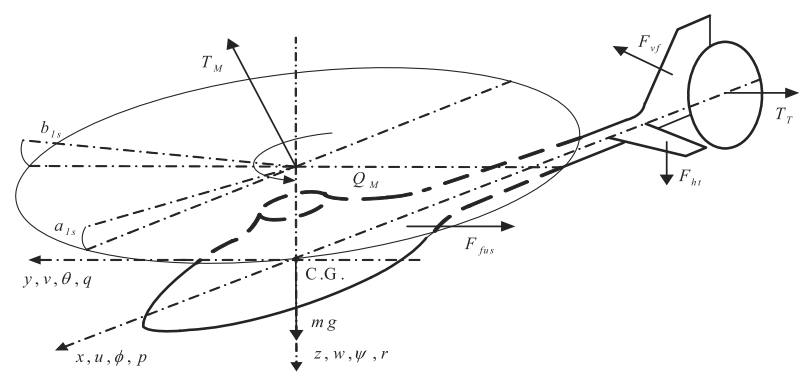

Fig. 3. Frames on an unmanned helicopter.

The nose, right side and downward directions of the helicopter are defined as the $x, y$ and $z$ axes of the body frame, respectively, with the origin located at the center of gravity. The 6-DOF dynamic model of the helicopter can be expressed by the following Newton-Euler equations 
with respect to the body frame (Cai, Chen, Dong and Lee, 2011a):

$$
\left\{\begin{array}{l}
m \dot{V}^{B}+\Omega^{B} \times m V^{B}=F_{\mathrm{ext}}^{B}, \\
I \dot{\Omega}^{B}+\Omega^{B} \times m \Omega^{B}=M_{\mathrm{ext}}^{B},
\end{array}\right.
$$

where the label $B$ denotes that the variable is defined in the body frame, $x$ denotes the cross-product of two vectors, $m$ is the mass of the helicopter, $I$ is the matrix of the moments of inertia, $V^{B}=\left[v_{b, x}, v_{b, y}, v_{b, z}\right]^{T}$ is the velocity vector, $\Omega^{B}=[p, q, r]^{T}$ is the angular velocity vector. $F_{\mathrm{ext}}^{B}$ and $M_{\mathrm{ext}}^{B}$ are the sums of external forces and torques, respectively. The force and torque generated by the fuselage, the horizontal stabilizer and the vertical stabilizer are ignored for simplification since they are quite small compared with those of the main rotor and tail rotor, especially in low speed flight.

Simplified $F_{\text {ext }}^{B}$ and $M_{\mathrm{ext}}^{B}$ are given as follows (He and Han, 2010):

$$
\begin{gathered}
F_{\mathrm{ext}}^{B}=\left[\begin{array}{c}
X_{M} \\
Y_{M}+Y_{T} \\
Z_{M}
\end{array}\right]+R_{H P \rightarrow B}\left[\begin{array}{c}
0 \\
0 \\
m g
\end{array}\right], \\
M_{\mathrm{ext}}^{B}=\left[\begin{array}{c}
L_{M}+Y_{M} h_{M}+Z_{M} y_{M}+Y_{T} h_{T} \\
M_{M}+M_{T}-X_{M} h_{M}+Z_{M} l_{M} \\
N_{M}-Y_{M} l_{M}-Y_{T} l_{T}
\end{array}\right],
\end{gathered}
$$

where $\left[X_{M}, Y_{M}, Z_{M}\right]^{T}$ and $\left[L_{M}, M_{M}, N_{M}\right]^{T}$ denote the forces and torques generated by the main rotor in the $x, y$ and $z$ axes, respectively, $\left[X_{T}, Y_{T}, Z_{T}\right]^{T}$ and $\left[L_{T}, M_{T}, N_{T}\right]^{T}$ denote the forces and torques generated by the tail rotor in the $x, y$ and $z$ axes, respectively, $\left[l_{T}, y_{T}, h_{T}\right]^{T}$ and $\left[l_{M}, y_{M}, h_{M}\right]^{T}$ are the distances between the center of gravity and the center of the main rotor or the tail rotor in the body frame, respectively. $R_{H P \rightarrow B}$ is the transformation matrix from the local horizontal plane coordinate frame to body coordinate frame.

Given the control input $\left[\theta_{M}, \theta_{\text {lat }}, \theta_{\text {lon }}, \theta_{T}\right]$, the corresponding control input exerting on the main rotor and tail rotor is $\left[\theta_{M}, a_{1 s}, b_{1 s}, \theta_{T}\right]$, where $a_{1 s}$ and $b_{1 s}$ are the tip-path-plane (TPP) flapping angles of the main rotor corresponding to $\theta_{\text {lat }}$ and $\theta_{\text {lon. The force }} T_{M}$ and the torque $Q_{M}$ generated by the main rotor can be calculated by $\theta_{M}$. The force $T_{T}$ and the torque $Q_{T}$ generated by the tail rotor can be calculated by $\theta_{T}$. The calculation of $T_{M}, Q_{M}, T_{T}$, and $Q_{T}$ will be ignored for simplification since they are related to the complicated aerodynamics of rotor; details about the calculation are given by $\mathrm{He}$ and Han (2010).

Given $T_{M}, Q_{M}, T_{T}$, and $Q_{T}$, the force and torque elements exerted on each axis are given as follows (He and Han, 2010):

$$
\left\{\begin{array}{l}
X_{M}=-T_{M} \sin a_{1 s}, \\
Y_{M}=T_{M} \sin b_{1 s}, \\
Z_{M}=-T_{M} \cos a_{a 1 s} \cos b_{1 s}, \\
L_{M}=-b_{1 s} k_{b 1 s}-Q_{M} \sin a_{1 s}, \\
M_{M}=-a_{1 s} k_{a 1 s}-Q_{M} \sin b_{1 s}, \\
N_{M}=-Q_{M} \cos a_{1 s} \cos b_{1 s}, \\
Y_{T}=-T_{T}, \\
M_{T}=-Q_{T},
\end{array}\right.
$$

where $k_{b 1 s}$ and $k_{a 1 s}$ are the rotor stiffness factors in the lateral and longitudinal axes, respectively; explicit coefficients are determined for a given helicopter and can be found in the work of He and Han (2010).

There are two frames used in the helicopter description: the body coordinate frame and the inertial coordinate frame. The kinematics model is defined as (Cai et al., 2011b)

$$
\begin{gathered}
{\left[\begin{array}{c}
\dot{\phi} \\
\dot{\theta} \\
\dot{\psi}
\end{array}\right]=\left[\begin{array}{ccc}
1 & s_{\phi} t_{\theta} & c_{\phi} t_{\theta} \\
0 & c_{\phi} & -s_{\phi} \\
0 & s_{\phi} / c_{\theta} & c_{\phi} / c_{\theta}
\end{array}\right]\left[\begin{array}{l}
p \\
q \\
r
\end{array}\right],} \\
\dot{P}_{n}=V_{n}=R_{i 2 b}^{-1} V_{b}, \\
R_{i 2 b}=\left[\begin{array}{ccc}
c_{\theta} c_{\psi} & c_{\theta} s_{\psi} & -s_{\theta} \\
s_{\phi} s_{\theta} c_{\psi}-c_{\phi} s_{\psi} & s_{\phi} s_{\theta} s_{\psi}+c_{\phi} c_{\psi} & s_{\phi} c_{\theta} \\
c_{\phi} s_{\theta} c_{\psi}+s_{\phi} s_{\psi} & c_{\phi} s_{\theta} s_{\psi}-s_{\phi} c_{\psi} & c_{\phi} c_{\theta}
\end{array}\right],
\end{gathered}
$$

where $s_{x}$ denotes $\sin (x), c_{x}$ denotes $\cos (x), t_{x}$ denotes $\tan (x) ; \phi, \theta$ and $\psi$ denote roll, pitch and yaw, respectively; $P_{n}$ and $V_{n}$ are the position and velocity vectors in the inertial coordinate frame.

A combined dynamic model can then be organized as Eqn. (7), with the state, input and measurement as follows:

- flight state:

$x_{k}=\left[\mathbf{V}_{b}, \mathbf{A}_{b}, \phi, \theta, \psi, p, q, r\right]^{T}$,

- actuator health coefficients:

$\theta_{k}=\left[\gamma_{1}, \gamma_{2}, \gamma_{3}, \gamma_{4}, \delta_{1}, \delta_{2}, \delta_{3}, \delta_{4}\right]$,

- actuator control input: $u_{k}=\left[\theta_{M}, \theta_{\mathrm{lat}}, \theta_{\mathrm{lon}}, \theta_{T}\right]^{T}$,

- measurement: $y_{k}=\left[\mathbf{V}_{b}, \mathbf{A}_{b}, \phi, \theta, \psi, p, q, r\right]^{T}$,

where $u_{k}$ is the flight controller's output which can be derived directly by the on-board processor. The measurement $y_{k}$ can be obtained by the UH's on-board navigation system. The UH's on-board sensors mainly include a GPS, an inertial navigation unit, a barometer and a magnetometer (Wu et al., 2010; Dai et al., 2012). Through the utilization of a differential GPS (DGPS) and the fiber optic gyroscope, the navigation system's accuracy can be greatly improved as show in Table 1 (this is mainly used for experimental validation purposes). 
Table 1. Measurement accuracy of the UH navigation system.

\begin{tabular}{|cc||cc|}
\hline Position & $0.02 \mathrm{~m}$ & Velocity & $0.02 \mathrm{~m} / \mathrm{s}$ \\
\hline Altitude & $0.02 \mathrm{~m}$ & Acceleration & $0.03 \mathrm{~m} / \mathrm{s}^{2}$ \\
\hline Attitude & $0.05^{\circ}$ & Angular rate & $0.1^{\circ} / \mathrm{s}$ \\
\hline
\end{tabular}

\section{Simultaneous state and parameter estimation methods}

With the combined dynamic model established in Section 3, the actuator FDD problem is transformed into a nonlinear state estimation problem. Then a key issue for actuator FDD is to select a suitable estimation method from the various existing estimation techniques. In the remainder of this paper, the main focus will be on the comparison of these different methods. Based on the way of representing measurement and system noise, the estimation methods included in this paper can be categorized into two groups: Kalman filter based estimations methods and set-membership based estimation methods. They are different in the assumption, presentation and propagation of noise over the system dynamic model.

4.1. Kalman filter based approach. The Kalman filter is the most well-known sequential estimation method for linear systems; the EKF applies the standard linear Kalman filter to nonlinear systems by linearization of nonlinear equations. This linearization in the EKF will introduce substantial errors into the estimation result, which may lead to poor performance or even the divergence of the estimation result.

4.1.1. Unscented Kalman filter. Since the commonly used EKF only retains the first-order Taylor series expansion of a nonlinear system, the performance of the EKF will deteriorate significantly with an increase in the system nonlinearity. For better estimation performance, the unscented Kalman filter was proposed to improve the accuracy, consistency and efficiency of the Kalman filter. The UKF uses a set of sigma points to represent the distribution of random variables; the sigma points are then propagated through the nonlinear dynamics, and the mean and covariance of the propagated random variable can be calculated using the propagated sigma points (Xiong et al., 2006; Kandepu et al., 2008; Julier and Uhlmann, 2004; Bätz et al., 2013).

A generally used sigma-point set to capture the random variable with mean value $\bar{x} \in \mathbb{R}^{n}$ and covariance matrix $P_{x}$ is defined as follows:

$$
\begin{aligned}
\chi_{0} & =\bar{x}, \\
w_{0}^{m} & =\frac{\lambda}{n+\lambda},
\end{aligned}
$$

$$
\begin{aligned}
\left.\chi_{i}\right|_{i=1, \ldots, n} & =\bar{x}+\left(\sqrt{(n+\lambda) P_{x}}\right)_{i} \\
w_{i}^{m} & =\frac{1}{2(n+\lambda)}, \\
\left.\chi_{i}\right|_{i=n+1, \ldots, 2 n} & =\bar{x}-\left(\sqrt{(n+\lambda) P_{x}}\right)_{i}, \\
w_{i}^{m} & =\frac{1}{2(n+\lambda)},
\end{aligned}
$$

where $\lambda=n\left(\alpha^{2}-1\right), \alpha \in(0,1)$, is the factor to determine the distribution of the sigma point $\chi_{i}$ is a sigma point, $w_{i}^{m}$ is the corresponding weighting factor for mean calculation and

$$
\sum_{i=0}^{2 n} w_{i}^{m}=1
$$

To include prior knowledge of the distribution, the weighting factor for covariance calculation is slightly different with respect to the mean calculation:

$$
w_{0}^{c}=\frac{\lambda}{n+\lambda}+\left(1-\alpha^{2}+\beta\right), \quad w_{i}^{c}=\frac{1}{2(n+\lambda)},
$$

$\beta>0$. Here $\beta$ is generally chosen as $\beta=2$ for Gaussian distributions.

The update process for the UKF is the same as for the EKF, except that the mean and covariance update are replaced by the calculation of the mean and covariance of the propagated sigma points. The UKF has proved to be more accurate, less susceptible and much simpler to use than the EKF (Campbell et al., 2007). Detailed stability analysis of the UKF can be found in the work of Xiong et al. (2006). The time and measurement updates of the UKF are listed as follows (Van Der Merwe, 2004):

- time update equations:

$$
\left\{\begin{array}{l}
\chi_{k-1} \in S\left(\bar{x}_{k-1}, P_{k-1}\right), \\
\chi_{k \mid k-1}^{*}=f\left(\chi_{k-1}, u_{k}\right), \\
\bar{x}_{k \mid k-1}=\sum_{i=0}^{2 n} w_{i}^{m} \chi_{i, k \mid k-1}^{*}, \\
P_{k \mid k-1}=\sum_{i=0}^{2 n} w_{i}^{c}\left(\chi_{i, k \mid k-1}^{*}-\bar{x}_{k \mid k-1}\right)^{2}+Q
\end{array}\right.
$$

- measurement update equations:

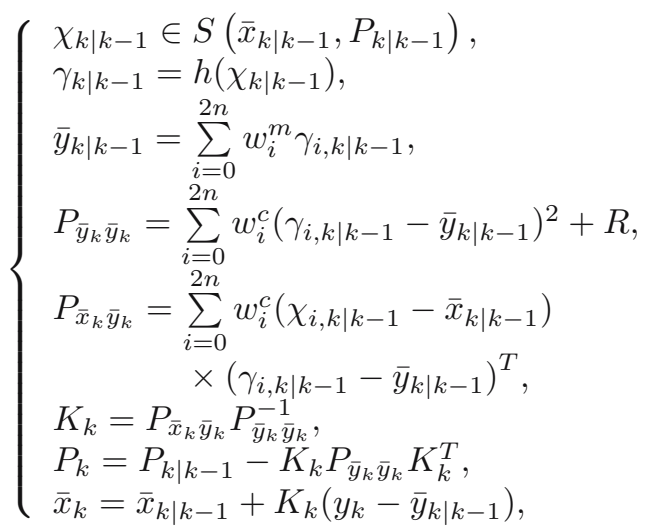


where $S\left(x_{k}, P_{k}\right)$ denotes sigma point calculation with mean value $x_{k}$ and covariance matrix $P_{k} . Q$ and $R$ are the covariance matrices of process noise and measurement noise, respectively. $Q$ and $R$ are normally set as constant before the implementation of the UKF.

If $Q$ and $R$ can be determined accurately, the UKF will accomplish optimal estimation in a probabilistic sense. In the real case, $R$ is much easier to be determined since it just relies on the sensor's characteristics, while $Q$ is hard to determine not only because of the modeling error but also because of the time-varying characteristics of AHCs. An adaptive algorithm with the ability to update $Q$ in accordance with the time-varying process noise characteristics will dramatically increase the estimation accuracy. MIT-UKF and KF-UKF are proposed for this purpose.

4.1.2. MIT rule based adaptive unscented Kalman filter. The MIT rule (also known as the gradient rule) was originally introduced for self-optimization adaptive control (Åström and Wittenmark, 2013). Here an MIT rule based adaptive algorithm will be introduced for system noise covariance matrix on-line update. The algorithm should find a loss function $J_{k}$ that is associated with the time-varying process noise covariance matrix $Q_{k}$.

In MIT-UKF, $J_{k}$ is defined as

$$
\left\{\begin{aligned}
e_{k}= & y_{k}-\bar{y}_{k \mid k-1} \\
S_{k}= & \frac{1}{N} \sum_{i=k-N+1}^{k} e_{k} e_{k}^{T} \\
\hat{S}_{k}= & \sum_{i=0}^{2 n}\left(\gamma_{i, k \mid k-1}-\bar{y}_{k \mid k-1}\right)\left(\gamma_{i, k \mid k-1}-\bar{y}_{k \mid k-1}\right)^{T} \\
& +R \\
J_{k}= & \operatorname{tr}\left[\left(S_{k}-\hat{S}_{k}\right)^{2}\right]
\end{aligned}\right.
$$

where $N$ is the window length used for accumulating prediction error $e_{k}$; a detailed definition of other variables in these equations can be found in Eqn. (20). The loss function is defined as the deviation of the time-averaged innovation covariance $S_{k}$ and the filter-computed innovation covariance $\hat{S}_{k}$. This loss function has proved to be more sensitive to the variation of process noise characteristics.

$Q_{k}$ is implicitly associated with $J_{k}$ through Eqn. (21), Based on the MIT rule, the update rule of $Q_{k}$ can be derived as follows:

$$
\left\{\begin{array}{l}
\dot{q}_{k}^{i}=-\eta \frac{\partial J_{k}}{\partial q_{k}^{i}}, \\
q_{k}^{i}=q_{k-1}^{i}-\dot{q}_{k}^{i} \Delta t,
\end{array}\right.
$$

where $q_{k}^{i}$ is the $i$-th diagonal element of $Q_{k}$ matrix at time $k, \eta$ is the tuning ratio which satisfies

$$
\eta_{k}>0, \quad \sum_{k} \eta_{k}=\infty, \quad \sum_{k} \eta_{k}^{2}<\infty
$$

$\Delta t$ is the discrete time step.

Through the recursive update process, $\hat{Q}_{k}$ will converge to the real $Q_{k}$ in a finite number of time steps, and with this accurate $Q_{k}$ the estimation accuracy of AHCs can be improved significantly compared with the traditional UKF. Detailed proofs of asymptotic behaviors and equations for optimization, which can be found in the work of Qi et al. (2007), will be ignored here for simplification.

\subsubsection{KF aided adaptive unscented Kalman fil-} ter (KF-UKF). As a different way of updating the process noise covariance matrix $Q_{k}$, the $\mathrm{KF}$ aided adaptive UKF uses an aided slave KF to estimate the noise covariance $Q_{k}$, and the standard UKF is executed using the recursively estimated noise covariance $Q_{k}$. Without modifications of the original UKF, the KF aided adaptive UKF is much simpler compared with the above-mentioned MIT-AUKF. The structure of KF-UKF is illustrated in Fig. 4

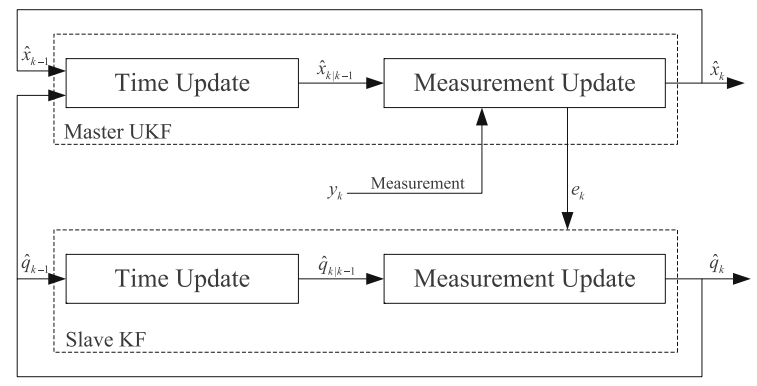

Fig. 4. KF-UKF structure.

With the assumption that the states are irrelevant, $Q_{k}$ is a diagonal matrix. Taking the elements of the principal diagonal matrix $Q_{k}$ as the slave KF's state vector, $q_{k}=\operatorname{vdiag}\left(Q_{k}\right)$, where $\operatorname{vdiag}(\cdot)$ denotes the vector of principal diagonal elements. The process model of $q_{k}$ is defined as a random walk dynamic model:

$$
q_{k}=q_{k-1}+w_{q, k}
$$

where $w_{q, k} \in \mathbb{R}^{n}$ is the process noise which is assumed to be a Gaussian white noise.

The measurement vector is defined as follows:

$$
S_{q}=\operatorname{vdiag}\left(\frac{1}{N} \sum_{i=k-N+1}^{k} e_{k} e_{k}^{T}\right) .
$$


In the UKF, $P_{k}$ 's measurement update rule is defined as

$$
\left\{\begin{array}{l}
P_{k}=P_{k \mid k-1}-K_{k} P_{\bar{y}_{k} \bar{y}_{k}} K_{k}^{T} \\
P_{k \mid k-1}=\sum_{i=0}^{2 n} w_{i}^{c}\left(\chi_{i, k \mid k-1}^{*}-\bar{x}_{k \mid k-1}\right)^{2}+Q_{k} .
\end{array}\right.
$$

Then the observation equation can be defined as

$$
S_{q}=\operatorname{vdiag}\left(P_{\bar{y}_{k} \bar{y}_{k}}\right)=q_{k} H_{P_{k}}+B_{P_{k}},
$$

where $B_{P_{k}}$ is a vector and $H_{P_{k}}$ is a matrix, both updated by the covariance matrix $P_{k}$ recursively. A detailed description of $B_{P k}$ and $H_{P k}$ is given by Qi et al. (2008; 2012).

The process and measurement model of $q_{k}$ can be established as follows:

$$
\left\{\begin{array}{l}
q_{k+1}=q_{k}+w_{q, k}, \\
S_{q}=q_{k} H_{P_{k}}+B_{P_{k}} .
\end{array}\right.
$$

The KF can be utilized based on this model for $q_{k}$ estimation recursively; the UKF will then take $Q_{k}=$ $\operatorname{diag}\left(q_{k}\right)$ as the process noise covariance matrix in the UKF process update step. The estimated $Q_{k}$ can converge to the real $\hat{Q}_{k}$, with a slower convergence rate compared with MIT-UKF (Qi et al., 2008).

4.2. Set-membership based approach. Set-membership based estimation represents the random variable as a set with the true value in it. There exist many kinds of description for this set, such as ellipsoid, orthotope, zonotope (Ingimundarson et al., 2009) and paralleltope (Zhou et al., 2008). Here the algorithms are based on an ellipsoid description which demands fewer parameters to represent the feasible set, with some very extraordinary characteristics, such as being more insightful for analogizing the covariance, invariant to linear transformations, and convenient for optimization (Zhou et al., 2008). Since a random variable with a bound is more reasonable compared with Gaussian approximation, set-membership based estimation is supposed to be more practical in real applications (Kotecha and Djuric, 2003; Campbell et al., 2007).

4.2.1. Extended set-membership filter. An ellipsoidal set description is defined as (Zhou et al., 2008)

$$
E(\bar{x}, P)=\left\{x \in \mathbb{R}^{n} \mid(x-\bar{x})^{T} P^{-1}(x-\bar{x}) \leq 1\right\},
$$

where $\bar{x}$ is the center of the ellipsoid set, $P$ is a positive-definite matrix representing the shape of the ellipsoid set. In order to propagate the ellipsoid set, we need to linearize the nonlinear system equation around $\bar{x}_{k}$ :

$$
\begin{aligned}
x_{k+1}= & \left.f(x)\right|_{x=\bar{x}_{k}}+\left.\frac{\partial f(x)}{\partial x}\right|_{x=\bar{x}_{k}}\left(x-\bar{x}_{k}\right) \\
& +\Delta_{f}+\omega_{k}, \\
\Delta_{f}= & \left.\left(x-\bar{x}_{k}\right)^{T} \frac{\partial f^{2}(x)}{\partial x^{2}}\right|_{x=\tilde{x}_{k}}\left(x-\bar{x}_{k}\right),
\end{aligned}
$$

where $\Delta_{f}$ is the linearization error, $\tilde{x}_{k} \in E\left(\bar{x}_{k}, P_{k}\right)$ and $\tilde{x}_{k}$ is chosen as the value to get the maximum $\Delta_{f}$. In the ESMF, process noise $w_{k}$ and measurement noise $v_{k}$ are assumed to obey $w_{k} \in E\left(0, Q_{k}\right)$ and $v_{k} \in E\left(0, R_{k}\right)$. Through interval analysis we can derive the linearization error bound $\bar{Q}_{k}$, and update the process noise bound as $\hat{Q}_{k}=Q_{k} \cup \bar{Q}_{k}$. In the same way, the measurement noise bound can be revised as $\hat{R}_{k}=R_{k} \cup \bar{R}_{k}$.

The estimation procedure is defined as follows (Zhou et al., 2008):

- time update equations:

$$
\left\{\begin{array}{l}
\bar{x}_{k, k-1}=f\left(\bar{x}_{k-1}\right), \\
A_{k}=\left.\frac{\partial f(x)}{\partial x}\right|_{x=\bar{x}_{k-1}}, \\
\beta_{k}=\frac{\sqrt{\operatorname{tr}\left(\hat{Q}_{k}\right)}}{\sqrt{\operatorname{tr}\left(\hat{Q}_{k}\right)}+\sqrt{\operatorname{tr}\left(A_{k} P_{k} A_{k}^{T}\right)}}, \\
P_{k, k-1}=A_{k} \frac{P_{k}}{1-\beta_{k}} A_{k}^{T}+\frac{\hat{Q}_{k}}{\beta_{k}},
\end{array}\right.
$$

- measurement update equations:

$$
\left\{\begin{array}{l}
C_{k}=\left.\frac{\partial h(x)}{\partial x}\right|_{x=\bar{x}_{k, k-1}}, \\
\rho_{k}=\frac{\sqrt{\max \left(\operatorname{eig}\left(\hat{R}_{k}\right)\right)}}{\sqrt{\max \left(\operatorname{eig}\left(C_{k} P_{k, k-1} C_{k}^{T}\right)\right)}+\sqrt{\max \left(\operatorname{eig}\left(\hat{R}_{k}\right)\right)}}, \\
W_{k}=C_{k} \frac{P_{k, k-1}}{1-\rho_{k}} C_{k}^{T}+\frac{\hat{R}_{k}}{\rho_{k}}, \\
K_{k}=\frac{P_{k, k-1} C_{k}^{T} W_{k}^{-1},}{1-\rho_{k}} \\
\bar{x}_{k}=\bar{x}_{k, k-1}+K_{k}\left[y_{k}-h\left(\bar{x}_{k, k-1}\right)\right], \\
\hat{P}_{k}=\frac{P_{k, k-1}}{1-\rho_{k}}-\frac{P_{k, k-1}}{1-\rho_{k}} C_{k}^{T} W_{k}^{-1} C_{k} \frac{P_{k, k-1}}{1-\rho_{k}}, \\
\delta_{k}=1-\left[y_{k}-h\left(\bar{x}_{k, k-1}\right)\right]^{T} W_{k}^{-1}\left[y_{k}-h\left(\bar{x}_{k, k-1}\right)\right], \\
P_{k}=\delta_{k} \hat{P}_{k} .
\end{array}\right.
$$

In the regular ESMF update process, $Q_{k}$ and $R_{k}$ are assumed to be constant. As mentioned in Section 4.2.1, $Q_{k}$ is not suitable to be considered a constant because of the modeling error as well as the time-varying 
characteristics of AHCs. An adaptive algorithm, which can update $Q_{k}$ on-line, will improve the estimation accuracy of AHCs. An MIT-ESMF is proposed for this purpose.

4.2.2. MIT rule based adaptive extended setmembership filter. The same updating MIT rule is adapted for $Q_{k}$ in MIT-ESMF as in MIT-UKF. Since the propagation of a random variable is done by the ellipsoid set process, a new loss function $J_{k}$ needs to be defined which is different from $J_{k}$ in Eqn. (21).

The loss function used in MIT-ESMF is defined as follows (Song et al., 2012):

$$
J_{k}=\left(1-\delta_{k}\right) \operatorname{tr}\left\{P_{k}\right\} .
$$

As shown in Eqn. (32), $\left(1-\delta_{k}\right)$ is the representation of the deviation of the measurement output from the estimated output, $\operatorname{tr}\left\{P_{k}\right\}$ is the representation of the estimated state. The loss function $J_{k}$ is defined as shown in Eqn. (33) because the accurate estimation of $Q_{k}$ will minimize $\operatorname{tr}\left\{P_{k}\right\}$ and $\left(1-\delta_{k}\right)$ at the same time. Since $\delta_{k}<1$ and $\operatorname{tr}\left\{P_{k}\right\}>0, J_{k}$ is positive-definite.

The update rule of $Q_{k}$ is the same as for the MIT-UKF in Eqn. (22), except the detailed presentation of $\partial J_{k} / \partial q_{k}^{i}$ and the choice of the tuning ratio $\eta$. A detailed equation for optimization and the convergence proof of this update rule can be found in the work of Song et al. (2012).

\section{Simulation results}

The nonlinear estimation algorithms introduced in Section 4 will be implemented for simultaneous state and AHC estimation based on the unmanned helicopter dynamic and kinematic model introduced in Section 3. The scheme for actuator FDD was introduced in Section 2 . Here emphasis will be on the comparison of these algorithms with respect to the estimation accuracy.

5.1. Simulation conditions. The simulation platform is built in the Matlab/Simulink environment. Based on the UH's dynamic model established in Section 3, a linearized model is derived for the hovering condition, and a linear-quadratic regulator (LQR) is designed as the flight controller. With the LQR flight controller, the UH can finish the basic flight mission such as hovering at a fixed point or forward flight at a fixed speed. There is an additional actuator fault module which can be used to specify various kinds of actuator faults. The process and measurement noise can be added in a different manner to compare the performance of these algorithms. In such a way, we can establish a full simulation platform for actuator FDD and FTC to validate various FDD and FTC methods.
The variation in an elevator servo's AHCs $\left(\gamma_{e}\right.$ and $\left.\delta_{e}\right)$ is designed to simulate the corresponding actuator fault on the unmanned helicopter. Other actuator AHCs can be estimated in the same way and will not be detailed in the simulation. Since three-axis angular velocities can reflect the influence of the elevator servo completely and are independent of other flight states, $x=\left[p, q, r, \gamma_{e}, \delta_{e}\right]^{T}$ is selected as the system state, $y=[p, q, r]^{T}$ is selected as the measurement, and $u=\left[\theta_{M}, \theta_{\mathrm{lat}}, \theta_{\mathrm{lon}}, \theta_{T}\right]^{T}$ is selected as the system input.

5.2. Comparison of the UKF and the ESMF. A different representation of noise characteristics and a different way of handling noise in the estimation step are the main differences between the UKF and the ESMF. Here, in this simulation, comparison of their performances in different noise conditions is made.

Two types of process noise are added in the simulation: Gaussian noise and uniformly distributed noise. The bound of Gaussian noise is defined as the triple square root of the diagonal element of covariance matrix, which is referred to as a three-sigma bound. Uniformly distributed noise is designed in the same bound as Gaussian noise for comparison.

In this simulation, the $\mathrm{UH}$ is supposed to fly at a forward speed of $10 \mathrm{~m} / \mathrm{s}$. An abrupt actuator fault is designed at $10 \mathrm{~s}$ with $\gamma_{e}=0.5$ which means a $50 \%$ loss in the effectiveness of the actuator. Different noise signals are added: Gaussian noise is designed as $N\left(0,10^{-4}\right)$, and uniformly distributed noise is designed as $U(-0.03,0.03)$.

The estimation result is shown in Fig. 5. The bound of the UKF estimation result is defined as the triple square root of the diagonal element of the covariance matrix. The true value of $\gamma_{e}$ can be estimated by both the UKF and the ESMF. The ESMF estimation shifts result the near the true value but with the true value lying on the estimation bound. Since $\hat{x}$ is just the center of the ellipsoidal set in the ESMF, the ESMF has no comparability with the UKF the with respect to the estimation accuracy. On the other hand, the ESMF's performance is almost the same under these two kinds of noise, but the UKF performance is quite different between these two kind of noise. This demonstrates that the system with bounded noise characteristics is suitable to apply the ESMF, and the system with Gaussian noise is suitable to apply the UKF.

The forward speed and the corresponding elevator control input are shown in Fig. 6 Because LQR can partly compensate for such a kind of actuator fault, the forward speed can be finally maintained at $8 \mathrm{~m} / \mathrm{s}$. A specified FTC would be needed to guarantee the flight performance and keep the forward speed at $10 \mathrm{~m} / \mathrm{s}$.

In another simulation, a sine actuator fault is designed at $10 \mathrm{~s}$ with $\delta_{e}=0.02 \sin (10(t-10) / 2 \pi)$, 


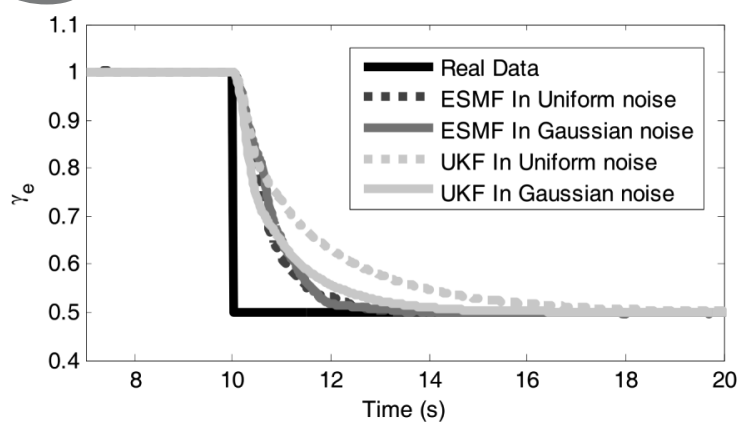

(a)

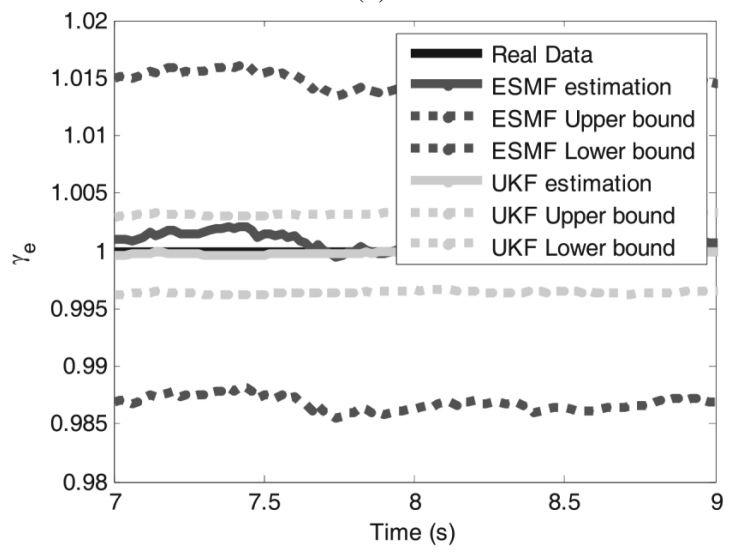

(b)

Fig. 5. Comparison of the UKF and the ESMF: estimation results for different noise characteristics (a), estimation of bounds (b).
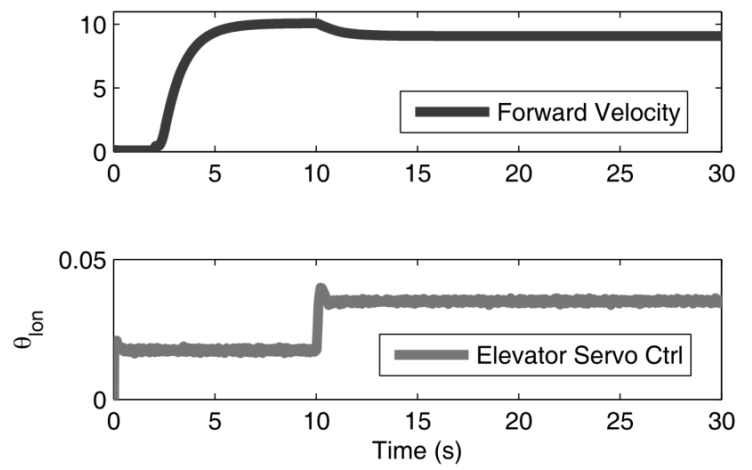

Fig. 6. System response to an actuator fault.

which means a sine bias of the actuator. The estimation result is shown in Fig. 7. Since we assume AHCs to be a slow-changing random-walk variable that cannot describe the sine actuator fault, the estimated $\hat{\delta}_{e}$ is in a phase delay and with a magnitude decrease compared with real $\delta_{e}$.

5.3. Comparison of MIT-ESMF, MIT-UKF and KFUKF. Because Kalman filter based methods and set membership based methods are quite different with their own advantages, as shown in Fig. 5 the performance of

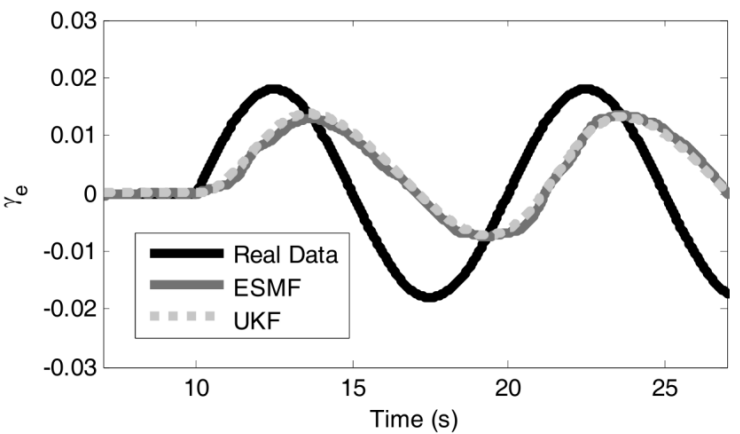

Fig. 7. Estimation result under a sine actuator fault of $\delta_{e}$.

MIT-UKF, KF-UKF and MIT-ESMF will not be compared in the following.

In the first simulation, the $\mathrm{UH}$ is supposed to fly at a forward speed of $10 \mathrm{~m} / \mathrm{s}$. An abrupt actuator fault is designed at $10 \mathrm{~s}$ with $\gamma_{e}=0.5$. Gaussian noise is added for the comparison of the UKF, KF-UKF and MIT-UKF. The noise is designed as $N\left(0,10^{-4}\right)$ firstly, with an abrupt change of $N\left(0,10^{-3}\right)$ at $20 \mathrm{~s}$ to validate the UKF, $\mathrm{KF}-\mathrm{UKF}$ and KF-UKF performance under time-varying noise conditions.

The results are shown in Fig. 8. At the beginning, the estimation results are almost the same with proper selection of $Q_{k}$; as the actuator fault occurred at $10 \mathrm{~s}$, MIT-UKF and KF-UKF can update the $Q_{k}$ to quickly follow the real $\gamma_{e}$, and MIT-UKF has a faster convergence rate compared with KF-UKF, the UKF can follow the real $\gamma_{e}$ very slowly. As the $Q_{k}$ changed at $20 \mathrm{~s}$, MIT-UKF and KF-UKF can update the real $Q_{k}$ to derive a more accurate estimation of $\gamma_{e}$ compared with the UKF.

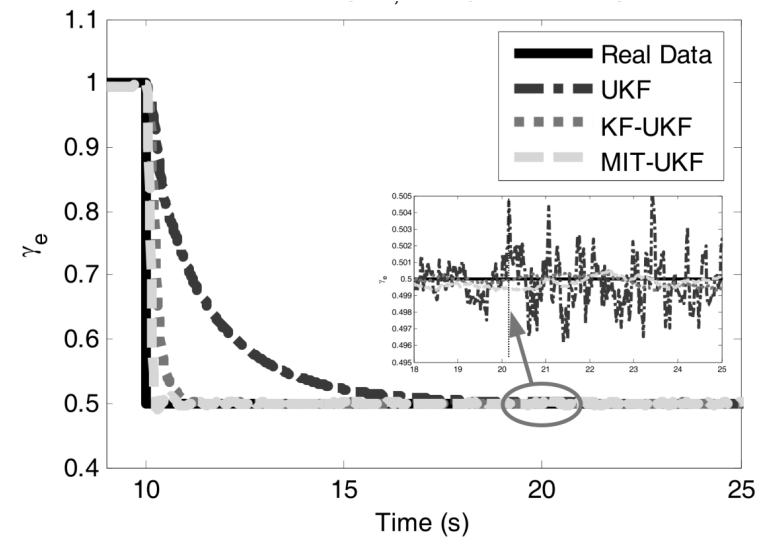

Fig. 8. Comparison of the UKF, MIT-UKF and KF-UKF under an abrupt change in $\gamma_{e}$ and time-varying process noise.

Uniformly distributed noise is added for the comparison of the ESMF and MIT-ESMF. The noise is designed as $U(-0.03,0.03)$ firstly, with an abrupt change characterized by $U(-0.06,0.06)$ at $20 \mathrm{~s}$ to validate the 
MIT-ESMF performance under time-varying noise. The estimation result is shown in Fig. 9. Better performance can be obtained for MIT-ESMF with the bound to the state estimated adaptively on-line.

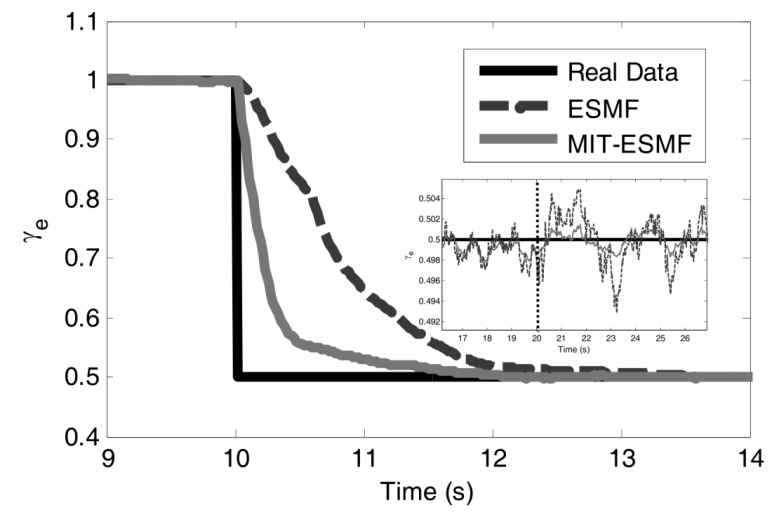

Fig. 9. Comparison of the ESMF and MIT-ESMF under an abrupt change in $\gamma_{e}$.

In the second simulation, an abrupt actuator fault is designed at $10 \mathrm{~s}$ with $\delta_{e}=0.02$. The corresponding estimation result is shown in Fig. 10. The computational

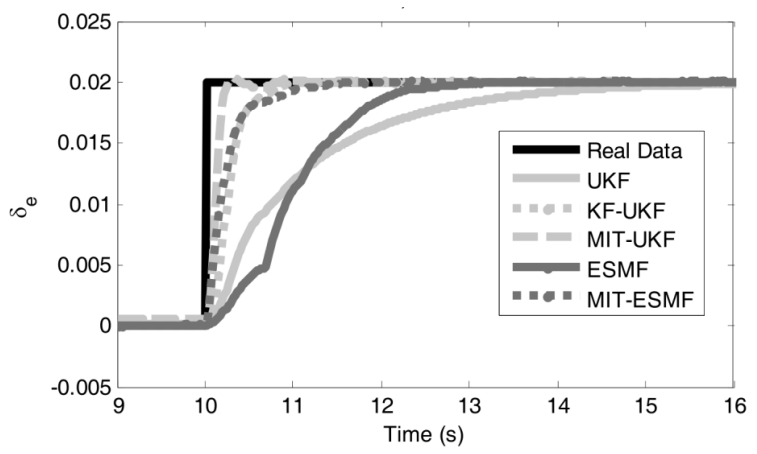

Fig. 10. Estimation result under an abrupt change of $\delta_{e}$ and time-varying process noise.

time for these algorithms is shown in Table 2. The adaptive schemes can make estimation of AHCs more accurate and faster at the expense of execution time.

Table 2. Time of computations for the whole simulation with 2000 steps.

\begin{tabular}{|c|c|c|c|c|}
\hline UKF & KF-UKF & MIT-UKF & ESMF & MIT-ESMF \\
\hline \hline $4.3 \mathrm{~s}$ & $4.7 \mathrm{~s}$ & $9.5 \mathrm{~s}$ & $5.2 \mathrm{~s}$ & $7.1 \mathrm{~s}$ \\
\hline
\end{tabular}

\section{Conclusion}

Simultaneous state and parameter estimation based fault detection and diagnosis for a $\mathrm{UH}$ was reviewed in this paper, which can transform the actuator FDD into a nonlinear estimation problem. Common nonlinear estimation methods, the UKF and the ESMF, were introduced firstly, followed with three adaptive nonlinear estimation methods, KF-UKF, MIT-UKF and MIT-ESMF, which were proposed by our team for the purpose of improving estimation accuracy and convergence rate. Based on the UH's dynamic model and an actuator fault model, a comparison of the different algorithms was made via simulation.

Simulation results demonstrate the effectiveness of the simultaneous state and parameter estimation approach in actuator FDD. Some conclusions can be made based on the simulation results: the ESMF can give an accurate bound for estimation which can further be used for robust controller design, and it can give an explicit indication of an actuator fault, which makes it superior to the UKF in fault detection; MIT-UKF is superior to KF-UKF in estimation accuracy but with longer execution times, and both of these two methods are significantly superior to the UKF in estimation accuracy; MIT-ESMF is superior to the ESMF with a fast convergence rate.

Even though the simulation results are encouraging, these estimation performances are still limited when implemented in real flight test validation. The main gaps between simulation and real flight tests include the modeling error and numerous disturbances exerted on the $\mathrm{UH}$ in real flight. These factors exceed the tolerable bound of the above mentioned estimation methods. In the next step, the estimation method should be improved towards the ability to manage strong modeling errors and disturbances.

\section{Acknowledgment}

This work was supported in part by the National Key Technology Research and Development Program of China under the grant 2013BAK03B01 and the National Natural Science Foundation of China under the grants 61433016 and 61203334 .

\section{References}

Amidi, O., Kanade, T. and Miller, J.R. (1998). Vision-based autonomous helicopter research at Carnegie Mellon Robotics Institute 1991-1997, Proceedings of the American Helicopter Society International Conference, Heli, Japan, pp. T7-3-1-T7-3-12.

Amoozgar, M.H., Chamseddine, A. and Zhang, Y. (2013). Experimental test of a two-stage Kalman filter for actuator fault detection and diagnosis of an unmanned quadrotor helicopter, Journal of Intelligent and Robotic Systems 70(4): 107-117.

Arne, W. and Jürgen, A. (2011). Robust fault isolation observers for non-square systems - a parametric approach, Proceedings of the 8th IFAC Symposium on Fault Detection, Su- 
pervision and Safety of Technical Processes, Mexico City, Mexico, pp. 1275-1280.

Åström, K.J. and Wittenmark, B. (2013). Adaptive Control, Courier Dover Publications, New York, NY.

Bätz, G., Weber, B., Scheint, M., Wollherr, D. and Buss, M. (2013). Dynamic contact force/torque observer: Sensor fusion for improved interaction control, The International Journal of Robotics Research 32(4): 446-457.

Bian, X., Li, X., Chen, H., Gan, D. and Qiu, J. (2011). Joint estimation of state and parameter with synchrophasors, Part II: Parameter tracking, IEEE Transactions on Power Systems 26(3): 1209-1220.

Cai, G., Chen, B., Dong, X. and Lee, T. (2011a). Design and implementation of a robust and nonlinear flight control system for an unmanned helicopter, Mechatronics 21(5): 803-820.

Cai, G., Chen, B. and Lee, T. (2011b). Unmanned Rotorcraft System, Springer-Verlag, London.

Campbell, M., Lee, J., Scholte, E. and Rathbun, D. (2007). Simulation and flight test of autonomous aircraft estimation, planning, and control algorithms, Journal of Guidance, Control, and Dynamics 30(6): 1597-1609.

Cui, N., Hong, L. and Layne, J. (2005). A comparison of nonlinear filtering approaches with an application to ground target tracking, Signal Processing 85(8): 1469-1492.

Dai, L., Qi, J., Wu, C. and Han, J. (2012). Magnetic compass error analysis and calibration for rotorcraft flying robot, Jiqiren (Robot) 34(4): 418-423, (in Chinese).

Drozeski, G., Saha, B. and Vachtsevanos, G. (2005). A fault detection and reconfigurable control architecture for unmanned aerial vehicles, Proceedings of the IEEE Aerospace Conference, Big City, MT, USA, pp. 1-9.

Ducard, G. and Geering, H. (2008). Efficient nonlinear actuator fault detection and isolation system for unmanned aerial vehicles, Journal of Guidance, Control, and Dynamics 31(1): 225-237.

He, Y. and Han, J. (2010). Acceleration-feedback-enhanced robust control of an unmanned helicopter, Journal of Guidance, Control, and Dynamics 33(4): 1236-1250.

Heredia, G., Ollero, A. and Bejar, M. (2008). Sensor and actuator fault detection in small autonomous helicopters, Mechatronics 18(2): 90-99.

Heredia, G., Remu, B. and Ollero, A. (2004). Actuator fault detection in autonomous helicopters, Proceedings of the 5th IFAC Symposium on Intelligent Autonomous Vehicles, Lisbon, Portugal, pp. 569-574.

Ingimundarson, A., Bravo, J., Puig, V., Alamo, T. and Guerra, P. (2009). Robust fault detection using zonotope-based set-membership consistency test, International Journal of Adaptive Control and Signal Processing 23(4): 311-330.

Johnson, E.N. and Schrage, D.P. (2003). The Georgia Tech unmanned aerial research vehicle: GTMax, Proceedings of the AIAA Guidance, Navigation, and Control Conference, Austin, TX, USA, pp. 11-14.
Julier, S. and Uhlmann, J. (2004). Unscented filtering and nonlinear estimation, Proceedings of the IEEE 92(3): 401-422.

Kandepu, R., Foss, B. and Imsland, L. (2008). Applying the unscented Kalman filter for nonlinear state estimation, Journal of Process Control 18(8): 753-768.

Kotecha, J. and Djuric, P. (2003). Gaussian particle filtering, IEEE Transactions on Signal Processing 51(10): 2592-2601.

Qi, J., Han, J. and Wu, Z. (2008). Rotorcraft UAV actuator failure estimation with KF-based adaptive UKF algorithm, Proceedings of the American Control Conference, Seattle, WA, USA, pp. 1618-1623.

Qi, J., Jiang, Z., Zhao, X. and Han, J. (2007). Adaptive UKF and its application in fault tolerant control of rotorcraft UAV, Proceedings of the AIAA Guidance, Navigation, and Control Conference, Hilton Head, SC, USA, pp. 43-57.

Qi, J., Song, D., Dai, L. and Han, J. (2009). Design, implement and testing of a rotorcraft UAV system, in T. Mung Lam (Ed.), Aerial Vehicles, InTech, Rijeka, pp. 537-554.

Qi, J., Song, D., Wu, C., Han, J. and Wang, T. (2012). KF-based adaptive UKF algorithm and its application for rotorcraft UAV actuator failure estimation, International Journal of Advanced Robotic Systems 9(132): 1-9.

Qi, J., Zhao, X., Jiang, Z. and Han, J. (2006). Design and implement of a rotorcraft UAV testbed, Proceedings of the IEEE International Conference on Robotics and Biomimetics, Kunming, China, pp. 109-114.

Qi, X., Qi, J., Theilliol, D., Zhang, Y. and Han, J. (2014). A review on fault diagnosis and fault tolerant control methods for single-rotor aerial vehicles, Journal of Intelligent and Robotic Systems 73(4): 535-555.

Qi, X., Theilliol, D., Qi, J., Zhang, Y., Han, J., Song, D., Wang, L. and Xia, Y. (2013). Fault diagnosis and fault tolerant control methods for manned and unmanned helicopters: A literature review, Proceedings of the International Conference on Control and Fault-Tolerant Systems (SysTol), Nice, France, pp. 132-139.

Song, D., Wu, C., Qi, J., Han, J. and Wang, T. (2012). A MIT-based nonlinear adaptive set-membership filter for the ellipsoidal estimation of mobile robots' states, International Journal of Advanced Robotic Systems 9(125): 1-12.

Van Der Merwe, R. (2004). Sigma-Point Kalman Filters for Probabilistic Inference in Dynamic State-Space Models, Ph.D. thesis, Oregon Health \& Science University, Portland, OR.

Wu, C., Song, D., Dai, L., Qi, J., Han, J. and Wang, Y. (2010). Design and implementation of a compact RUAV navigation system, Proceedings of the IEEE International Conference on Robotics and Biomimetics, Tianjin, China, pp. 1662-1667.

Wu, C., Song, D., Qi, J. and Han, J. (2012). Rotorcraft UAV actuator failure detection based on a new adaptive set-membership filter, Proceedings of the 5th International Conference on Intelligent Robotics and Applications, Montreal, Canada, pp. 433-442. 
Xiong, J. (2013). Set-Membership State Estimation and Application on Fault Detection, Ph.D. thesis, French National Center for Scientific Research, Toulouse.

Xiong, K., Zhang, H. and Chan, C. (2006). Performance evaluation of UKF-based nonlinear filtering, Automatica 42(2): 261-270.

Zhang, Y. and Jiang, J. (2008). Bibliographical review on reconfigurable fault-tolerant control systems, Annual Reviews in Control 32(2): 229-252.

Zhou, B. and Han, J. (2007). A comparison of nonlinear estimation methods for tracked vehicle with slipping, Proceedings of the IEEE International Conference on Control and Automation, Guangzhou, China, pp. 389-394.

Zhou, B., Han, J. and Liu, G. (2008). A UD factorization-based nonlinear adaptive set-membership filter for ellipsoidal estimation, International Journal of Robust and Nonlinear Control 18(16): 1513-1531.

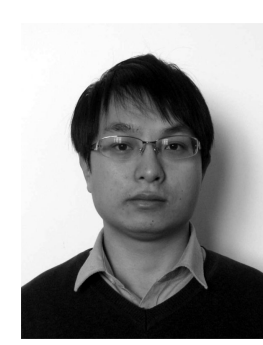

Chong Wu is a Ph. D. candidate at the Shenyang Institute of Automation, Chinese Academy of Sciences. He received his bachelor's degree from the Huazhong University of Science and Technology in 2008. His research interests include prediction and control for fly-robot with rotor wings.

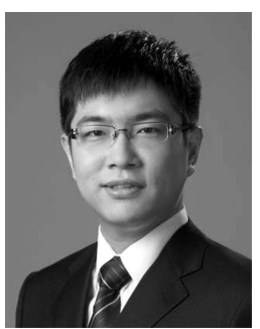

JunTong Qi is a professor at the Shenyang Institute of Automation, Chinese Academy of Sciences. He received his bachelor's degree from Tianjin University in 2004, and his doctoral degree from the Shenyang Institute of Automation in 2009. His research interests cover fault detection and fault tolerance control for fly-robot with rotor wings.

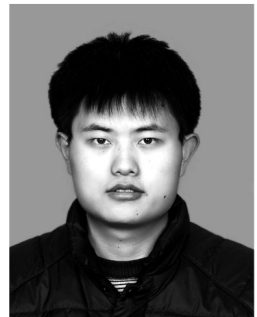

Xin Qi is Ph. D. candidate at the Shenyang Institute of Automation, Chinese Academy of Sciences. He received his bachelor's degree from Tianjin University in 2011. His research interests include fault detection and fault tolerance control for fly-robot with rotor wings.

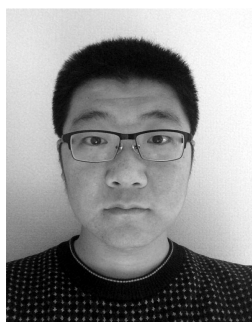

Dalei Song is an associate professor at the Shenyang Institute of Automation, Chinese Academy of Sciences. He received his bachelor's degree from Beihang University in 2006, and his doctoral degree from Shenyang Institute of Automation in 2011. His research interests cover modeling and control for fly-robot with rotor wings.

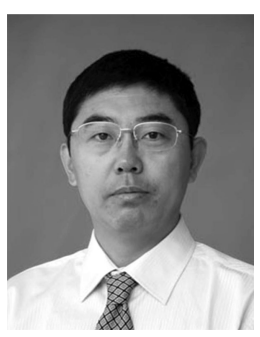

Jianda Han is a professor at the Shenyang Institute of Automation, Chinese Academy of Sciences. His research interests cover sensor based robot robust control system design, multi-robot system, and intelligent control system design.

Received: 18 February 2014

Revised: 6 May 2014

Re-revised: 12 August 2014 